\title{
FINITE ELEMENT ANALYSIS OF A FLOATING RECTANGULAR
}

\section{PLATE}

\author{
Bhagirathi Tripathy ${ }^{1}$, Pratap Kumar Pani ${ }^{2}$ \\ ${ }^{1}$ Lecturer, Department of Civil Engineering, IGIT, Sarang, Odisha, India \\ ${ }^{2}$ Professor, Department of Civil Engineering, IGIT, Sarang, Odisha, India
}

\begin{abstract}
The free vibration characteristics of a floating rectangular plate considering the fluid-structure interaction effects between the plate and the reservoir are studied. The fluid is assumed to be inviscid, incompressible and the reservoir bottom is assumed to be horizontal and rigid. Finite element technique is used to solve this interaction problem. The eigen frequencies of the plate are computed and the effects of the flexibility of the plate, depth of the reservoir are investigated.
\end{abstract}

Keywords -inviscid,irrotational,eigenfrequency,FEM,VLFS, hydroelasticity.

\section{INTRODUCTION}

With a growing population and the corresponding demand for settlement and need for basic and recreational facilities in land, countries with long coastlines, have resorted to land reclamation. But, huge cost, impact on coast lines, marine eco system and sometimes opposition from neighboring countries, reclamation is not feasible all the time. Hence, the concept of floating platforms came and has been successfully tried in many places. Today floating platforms are used for various purposes like exploration platforms, naval base, air strips, rescue bases, amusement parks and settlement colonies etc. A lot of research has been carried out. The earlier works like that of John [1] were based on the motion of rigid plates due to wave action. Later investigations were focused on the flexibility of the large floating plates. Thus, hydroelastic analysis took centre stage in the analysis of mat-like floating structures. Breakthrough works by Bishop and Price [2] and Price and Wu [3] led to the full 3D hydroelasticity theory, where the Green function method is used to model the fluid. The plate is modelled as an elastic thin plate with free edges. The fluid is incompressible, inviscid and its motion is irrotational so that a velocity potential exists. The amplitude of the incident wave and the motions of the VLFS are both small and only the vertical motion of the structure is considered.

Eigen frequencies of a plate either immersed or in contact with a fluid decrease significantly compared to those in vacuum, especially for the fundamental one. This is because the vibration of the plate is transferred to the fluid causing an increase in the kinetic energy of the surrounding fluid. Though exact solution for this type of interaction problem is difficult to obtain, there are many analytical approaches for plates of different shapes with some rational boundary conditions and approximations of the fluid domain. In the early phase of work on this area, Lamb (1920) studied the change in natural frequencies of a thin clamped circular plate in an aperture of an infinitely long plane rigid wall in contact with water. Later, Powell and Roberts (1923) [4] experimentally verified the work of Lamb and Mclachlan (1932)[5] extended Lamb's work to circular plates without any supports. Kwak and Kim (1991) [6] and later Kwak (1991) [7] obtained the non-dimensional added virtual mass incremental (NAVMI) factors for circular plates placed on a free liquid surface using Hankel's transformation for axisymmetric modes and all other modes. All these works are based on the assumption that mode shapes of the plate remain same both in contact with the fluid and in vacuum. Kwak (1996) [8] investigated the effect of water on mode shapes and observed that except the fundamental mode, other modes are influenced by the presence of water. The distortion of mode shapes increases with the increase in mode numbers. The plate was considered to be thin and made of isotropic, homogeneous and linearly elastic material. Kirchhoff's theory for plate vibration and analytical-Ritz method for fluid-structure interaction were adopted for the analysis.

For the solution of interaction problems, though analytical methods provide better accuracy, their use is limited to either very special or simple cases because of the mathematical complexities involved. However, due to the availability of high speed computational facilities, several numerical techniques may be adopted to obtain a meaningful solution of such complex problems. Among different numerical techniques used, finite element method (FEM) is mostly preferred due to its easy implementation in a wide range of problems.

The focus of the present work is to investigate the effect of the flexibility of the plate and fluid depth on eigen frequencies of the plate floating over the fluid. 


\section{APPLICATION OF RECTANGULAR PLATE AS}

\section{VLFS}

There are basically two types of very large floating structures (VLFSs), namely the semisubmersible- type and the pontoontype. Semi-submersible type floating structures are raised above the sea level using column tubes or ballast structural elements to minimize the effects of waves while maintaining a constant buoyancy force. Thus they can reduce the wave induced motions and are therefore suitably deployed in high seas with large waves. Floating oil drilling platforms used for drilling for and production of oil and gas are typical examples of semisubmersible-type VLFSs. When these semi-submersibles are attached to the seabed, using vertical tethers with high pretension as provided by additional buoyancy of the structure, they are referred to as tension-leg platforms. In contrast, pontoon-type floating structures lie on the sea level like a giant plate floating on water. Pontoon-type floating structures are suitable for use in only calm waters, often inside a cove or a lagoon and near the shoreline. Large pontoon-type floating structures have been termed Mega-Floats by Japanese engineers. As a general rule of thumb, Mega-Floats are floating structures with at least one of its length dimensions greater than $60 \mathrm{~m}$. Referring to Fig. 1, a Mega-Float system consists of a (a) very large pontoon floating structure, (b) mooring facility to keep the floating structure in place, (c) an access bridge or floating road to get to the floating structure from shore, and (d) a breakwater (usually needed if the significant wave height is greater than $4 \mathrm{~m}$ ) for reducing wave forces impacting the floating structure.

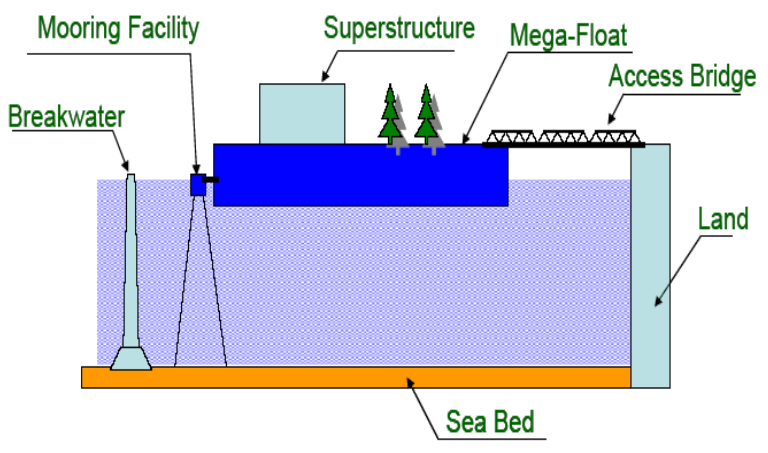

Fig-1 Components of Mega-Float System

\section{THE MODEL OF INVESTIGATION} (RECTANGULAR VLFS)

A rectangular plate of width $2 a$ and length $2 b$ is floating on a reservoir of infinite extent as shown in Fig. 1. The fluid is considered to be incompressible and inviscid with small amplitude motion. The effect of the static pressure is not considered in the analysis. It is assumed that the reservoir floor is horizontal and rigid. The plate is uniformly thick and its material is homogeneous, isotropic and linearly elastic in nature. In the analysis, the 3-D fluid domain is discretized considering eight noded brick elements and the plate using four noded quadrilateral plate elements. Mindlin's plate bending theory is used to analyze the plate.

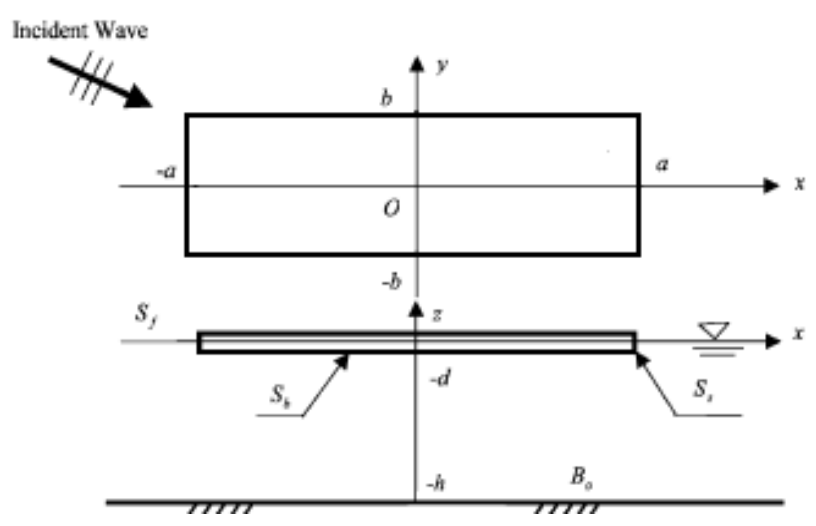

Fig-2 Pontoon type of VLFS under wave action

The fluid-structure system and the coordinate system are shown in Fig. 2. The origin of thecoordinate system is on the undisturbed free surface. The $\mathrm{z}$-axis is pointing upwards, andthe sea-bed is assumed to be flat at $\mathrm{z}=-\mathrm{h}$. The VLFS has a maximum length of $2 \mathrm{a}$ in the $\mathrm{x}$ direction, a maximum width of $2 \mathrm{~b}$ in the $\mathrm{y}$-direction, and $\mathrm{a}$ draft $\mathrm{d}$ in the $\mathrm{z}$-direction. Theproblem at hand is to determine the response of the VLFS under the action of wave forces.

\subsection{Basic Assumptions for Hydroelastic Analysis of VLFS}

In a basic hydroelastic analysis of pontoon-type VLFSs, the following assumptions are usually made:

- The VLFS is modeled as an elastic (isotropic/orthotropic) thin plate with free edges.

- The fluid is incompressible, inviscid and its motion is irrotational so that a velocity potential exists.

- The amplitude of the incident wave and the motions of the VLFS are both small and only the vertical motion of the structure is considered (i.e. we constrained the plate from moving horizontally in the analysis).

- There are no gaps between the VLFS and the free fluid surface.

The analysis may be carried out in the frequency domain or in the time domain. Most hydroelastic analyses are carried out in the frequency-domain, being the simpler of the two. However, for transient responses and for nonlinear equations of motion due to the effects of a mooring system or nonlinear wave (as in a severe wave condition), it is necessary to perform the analysis in the time-domain. Below, we present the governing equations, boundary conditions and briefly describe the commonly used methods for the analysis in the frequency-domain and in the time-domain. 


\section{GOVERNING EQUATIONS FOR THE PLATE STRUCTURE}

The un-damped free vibration of the gate structure is given as

$$
[M]\{\ddot{\bar{W}}\}+[K]\{\bar{W}\}=\{0\}
$$

Where, $[M]$ and $[K]_{\text {are the mass and stiffness matrices of the }}$ gate. $[\bar{W}]$ and $[\ddot{\bar{W}}]$ are the nodal displacement and acceleration vectors. In Mindlin's plate theory where shear deformations are taken into account, the nodal displacement is represented as

$$
[\bar{W}]=\left[\bar{W}_{z}, \theta_{x}, \theta_{y}\right]
$$

$\bar{W}_{z}$ is the transverse deflection along $\mathrm{Z}$ direction and $\theta_{x}, \theta_{y}$ are the rotations about $\mathrm{Y}$ and $\mathrm{Z}$ axes. The element stiffness matrix $[k]_{e}$ is split into flexure and shear parts to avoid shear locking. The corresponding split matrices are represented as

$$
\begin{aligned}
& {\left[k^{f}\right]_{e}=\int_{A} \frac{t_{h}^{3}}{12}\left[B^{f}\right]^{T}[D]\left[B^{f}\right] d A} \\
& {\left[k^{s}\right]_{e}=\int_{A} \kappa t_{h}\left[B^{s}\right]^{T}\left[D^{s}\right]\left[B^{s}\right] d A}
\end{aligned}
$$

Where, $[D] \&\left[D^{s}\right]$ are the constitutive matrices, $\left[B^{f}\right] \&\left[B^{s}\right]$ are the strain-displacement matrices for the flexure and the shear part respectively. The factor $\kappa$ depends on the shape of the cross section of the element and is $5 / 6$ for rectangular cross section as considered here.

Governing equation for the fluid domain considering non viscous incompressible fluid having irrotational motion is given as

$$
\nabla^{2} \mathrm{P}=0
$$

\section{BOUNDARY CONDITIONS}

For the problem under consideration of the floating plate, the following boundary conditions are adopted (Fig 2)

1. $p(x, y, 0)=0$ on $S_{f}$, where $h$ is the height of the free water surface above the reservoir bed.
2. $\frac{\partial p}{\partial n}(x, y,-h)=0$ on $S_{b}$, as the reservoir bed is horizontal and rigid.

3. $\frac{\partial p}{\partial n}(x, 0, z)=0, \frac{\partial p}{\partial n}(x, a, z)=0, \quad \frac{\partial p}{\partial n}(0, y, z)=$ $0, \frac{\partial p}{\partial n}\left(b, y, z 0=0\right.$ on $S_{s}, b$ is the width of the fluid domain considered for analysis.

\section{TIME -DOMAIN ANALYSIS}

The commonly-used approaches for the time-domain analysis of VLFS are the direct time integration method $[9,10]$ and the method that uses Fourier transform [11-15]. In the direct time integration method, the equations of motion are discretized for both the structure and the fluid domain. In the Fourier transform method, we first obtain the frequency domain solutions for the fluid domain and then Fourier transform the results for substitution into the differential equations for elastic motions. The equations are then solved directly in the time domain analysis by using the finite element method or other suitable computational methods.

\section{FINITE ELEMENT FORMULATION FOR THE FLUID DOMAIN}

The weighted integral form of eqn. 5 over an element is given as

$$
\int_{\Omega_{e}} \phi\left(\nabla^{2} p\right) d \Omega_{e}
$$

Where, $\phi$ is the weight function and $\Omega_{e}$ is the elemental volume. The pressure at any point inside the element is interpolated as

$$
p=\sum_{i=1}^{n} N_{i} \bar{p}_{i}
$$

$\bar{p}_{i}(\mathrm{i}=1,2,----\mathrm{n})$ is the nodal value of pressure at node $i, N_{i}$ is the interpolation function corresponding to the node and the summation is over all the number of nodes. Using the Galerkin's weighted residual method, the ith equation may be written as

$$
\int_{\Omega_{e}} N_{i}\left(\frac{\sum_{j=1}^{n} \partial^{2}\left(N_{j} \bar{p}_{j}\right)}{\partial x^{2}}+\frac{\sum_{j=1}^{n} \partial^{2}\left(N_{j} \bar{p}_{j}\right)}{\partial y^{2}}+\frac{\sum_{j=1}^{n} \partial^{2}\left(N_{j} \bar{p}_{j}\right)}{\partial z^{2}}\right) d \Omega_{e}=0
$$


Since, nodal pressure values are constant, taking them out of integration and integrating the rest with integration by parts, eqn. 8 may be represented as

$$
\begin{aligned}
& -\int_{\Omega_{\ell}}\left(\frac{\partial N_{i}}{\partial x} \sum_{j=1}^{n} \frac{\partial N_{j}}{\partial x}+\frac{\partial N_{j}}{\partial y} \sum_{j=1}^{n} \frac{\partial N_{j}}{\partial y}+\frac{\partial N_{i}}{\partial z} \sum_{j=1}^{n} \frac{\partial N_{j}}{\partial z}\right) S_{\varepsilon}\left[\bar{p}_{1} \ldots \bar{p}_{n}\right]^{T} \\
& +\int_{\Gamma_{e}} N_{i}\left(\frac{\partial p}{\partial q}\right) d \Gamma_{e}=0
\end{aligned}
$$

Where, $n_{i}$ is the normal to the surface and $\Gamma \mathrm{e}$ indicates integration over the surface only. Eqn. 9 may be expressed for the whole domain in matrix form as

$$
\begin{aligned}
& {[G]\{\bar{p}\}=\{Q\}} \\
& {[G]=\sum_{\Omega_{e}} \int_{e}\left(\frac{\partial[N]^{T}}{\partial x} \frac{\partial[N]}{\partial x}+\frac{\partial[N]^{T}}{\partial y} \frac{\partial[N]}{\partial y}+\frac{\partial[N]^{T}}{\partial z} \frac{\partial[N]}{\partial z}\right) d \Omega_{e}} \\
& {[Q]=\sum_{S} \int_{\Gamma_{e}}[N]^{T} \frac{\partial p}{\partial n} d \Gamma_{e}} \\
& {[N]=\left[N_{1}, N_{2},-----N_{n}\right]}
\end{aligned}
$$

$\{Q\}$ may be separated into different parts for different boundaries.

$$
\{Q\}=\left\{Q_{f}\right\}+\left\{Q_{f s}\right\}+\left\{Q_{b}\right\}+\left\{Q_{s}\right\}
$$

At the free surface $\left\{Q_{f}\right\}=0$

At the fluid structure interface

$$
\begin{aligned}
& \left\{Q_{f s}\right\}=-\rho_{f}\left[\boldsymbol{R}_{f s}\right]\{\overline{\boldsymbol{a}}\} \\
& {\left[\boldsymbol{R}_{f s}\right]=\sum_{S_{f s}} \int_{\Gamma_{e}}[N]^{T}[N] d \Gamma_{e}}
\end{aligned}
$$

At reservoir bottom surface

$$
\left[Q_{b}\right]=0
$$

On either side of the plate, the assumed boundary condition leads to

$$
\left\{Q_{s}\right\}=0
$$

Combining all these

$$
[G]\{\bar{p}\}=-\rho_{f}\left[R_{f s}\right]\{\bar{a}\}
$$

\section{COUPLED MOTION OF THE PLATE AND THE FLUID DOMAIN}

The boundary motion is prescribed by the movement of the plate. Replacing the acceleration $\{\bar{a}\}$ in eqn. 15 with the nodal acceleration of the gate

$$
Q_{f s}=[S]\left\{\ddot{w}_{e}\right\}
$$

Where

$$
[S]=\sum_{S_{f s}} \int_{\Gamma_{e}}[N]^{T} \rho_{f}[\bar{N}] d \Gamma_{e}
$$

$[N]$ is the interpolation function for pressure in the fluid domain and $[\bar{N}]$ is the interpolation function for nodal displacement of the plate. When the effect of dynamic pressure on the plate structure is considered, eqn 1 may be rewritten as

$$
[M]\{\ddot{\bar{W}}\}+[K]\{\bar{W}\}=\left\{\bar{f}_{s}\right\}
$$

Where, $\left\{\bar{f}_{s}\right\}$ is the forcing term due to pressure from the fluid at the interface.

$$
\left\{\bar{f}_{s}\right\}=-\int_{\Gamma}[\bar{N}]^{T} p d \Gamma=-\frac{1}{\rho_{f}}[S]^{T}\{\bar{p}\}
$$

As $p=[N][\bar{p}]$

Now, two coupled equations are obtained as

$$
[G]\{\bar{P}\}=\{Q\}=[S]\{\ddot{\bar{W}}\}
$$




$$
\begin{aligned}
& {[M]\{\dot{\bar{W}}\}+[K]\{\bar{W}\}+\frac{1}{\rho_{f}}[S]^{T}\{\bar{P}\}=\{0\}} \\
& \{\bar{P}\}=[G]^{-1}[S]\{\ddot{\bar{W}}\}
\end{aligned}
$$

So, eqn. 26 may be represented as

$$
[\bar{M}]\{\ddot{\bar{W}}\}+[K]\{\bar{W}\}=\{0\}
$$

where,

$$
[\bar{M}]=[M]+\frac{1}{\rho_{f}}[S]^{T}[G]^{-1}[S]
$$

The eigenfrequencies are evaluated from eqn. 28 using Jacobi's method. The non dimensional frequencies are represented as

$$
\Omega^{2}=\rho_{p} t_{h} \omega^{2} a^{4} / D_{p}
$$

Table-1: Eigenfrequencies of a plate of $1.0 \mathrm{~m} \times 1.0 \mathrm{~m} \times 0.02 \mathrm{~m}$ floating over water of depth $1.0 \mathrm{~m}$

\begin{tabular}{|l|l|l|l|l|l|}
\hline $\begin{array}{l}\text { Non } \\
\text { dimensional } \\
\text { frequency }\end{array}$ & $\Omega_{1}$ & $\Omega_{2}$ & $\Omega_{3}$ & $\Omega_{4}$ & $\Omega_{5}$ \\
\hline In vacuum & 3.70 & 9.12 & 24.60 & 28.83 & 35.86 \\
\hline $\begin{array}{l}\text { On floating } \\
\text { condition }\end{array}$ & 3.65 & 7.04 & 16.95 & 20.65 & 26.46 \\
\hline
\end{tabular}

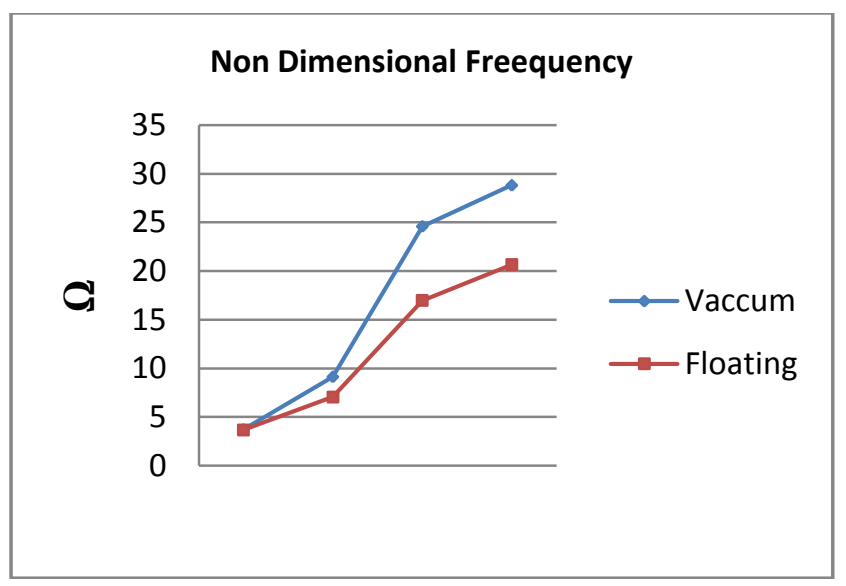

Fig-3 Non Dimensional Frequency
Table-2: Eigenfrequencies of a plate of $1.0 \mathrm{~m} \mathrm{x} 1.0 \mathrm{~m}$ for different thicknesses floating over water of depth $1.0 \mathrm{~m}$

\begin{tabular}{|l|l|l|l|l|l|}
\hline b/th & $\Omega_{1}$ & $\Omega_{2}$ & $\Omega_{3}$ & $\Omega_{4}$ & $\Omega_{5}$ \\
\hline 0.01 & 3.57 & 5.95 & 14.13 & 16.96 & 21.99 \\
\hline 0.02 & 3.65 & 7.04 & 16.95 & 20.65 & 26.46 \\
\hline 0.04 & 3.67 & 7.77 & 19.05 & 23.55 & 29.34 \\
\hline 0.06 & 3.67 & 7.93 & 19.71 & 24.50 & 29.56 \\
\hline 0.08 & 3.65 & 7.90 & 19.77 & 24.68 & 28.82 \\
\hline 0.10 & 3.60 & 7.78 & 19.49 & 24.46 & 28.82 \\
\hline
\end{tabular}

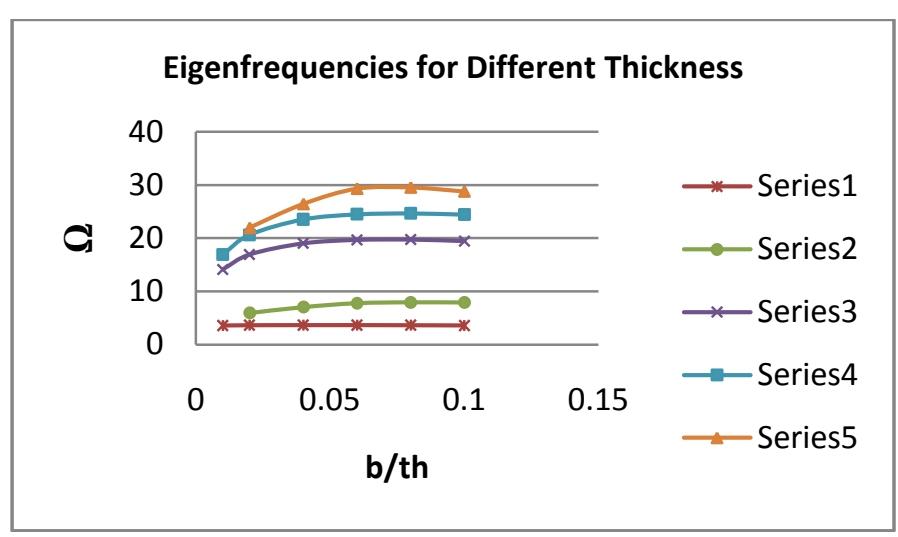

Fig-4 Eigenfrequencies for Different Thickness

Table-3: Eigenfrequencies of a plate of $1.0 \mathrm{~m} \times 1.0 \mathrm{~m} \times 0.02 \mathrm{~m}$ floating over water for different depth of the fluid

\begin{tabular}{|l|l|l|l|l|l|}
\hline $\begin{array}{l}\text { Depth of } \\
\text { fluid } \\
\text { In metre }\end{array}$ & $\Omega_{1}$ & $\Omega_{2}$ & $\Omega_{3}$ & $\Omega_{4}$ & $\Omega_{5}$ \\
\hline 1.0 & 3.65 & 7.04 & 16.95 & 20.65 & 26.46 \\
\hline 1.5 & 1.75 & 4.44 & 10.04 & 16.79 & 20.24 \\
\hline 2.0 & 0.99 & 3.05 & 5.89 & 11.67 & 15.44 \\
\hline 2.5 & 0.62 & 2.28 & 3.76 & 8.47 & 10.386 \\
\hline 3.0 & 0.44 & 1.77 & 2.77 & 6.30 & 8.02 \\
\hline
\end{tabular}

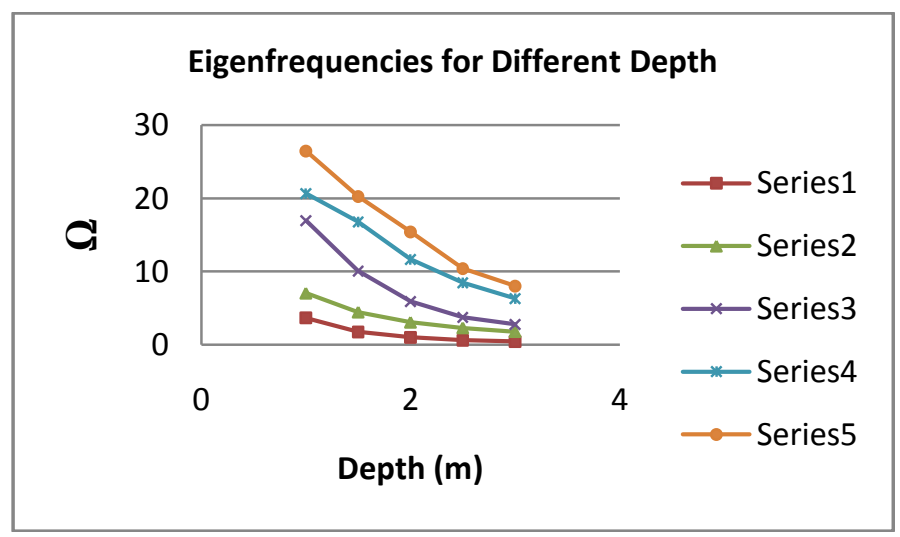

Fig-5 Eigenfrequencies for Different Depth 


\section{RESULTS AND DISCUSSION \& CONCLUSIONS}

The changes in eigenfrequencies due to the presence of the fluid is studied both for the flexibility of the plate and for the depth of the fluid. As shown in Figure-3, the eigenfrequencies decrease because of the presence of the fluid. The effect of the flexural rigidity of the plate is investigated by observing the reduction for different thicknesses of the plate. As observed from Figure-4, reduction of the eigenfrequencies increases with increase in flexibility of the plate. Depth of the fluid domain plays an important role with eigenfrequencies decreasing with the depth of the fluid as shown in Figure-5. The advanced research in this field will definitely will bring a revolution regarding the acute land problem and other disaster management problem.

\section{REFERENCES}

[1]. John F. On the motion of floating bodies. ommunications on Pure and Applied Mathematics, Part I: 1949;2:13-57; Part II: $1950 ; 3: 45-100$.

[2]. Bishop RED, Price WG" An introduction to ship hydroelasticity" , Journal of Sound and Vibration, Volume87, Issue 3, 8 April 1983, Pages 391-407.

[3]. Price WG, Wu Y. In: Niordson FI, Olhoff N, editors. Hydroelasticity of marine structures, theoretical and applied mechanics Elsevier Science Publishers; 1985, pp. 311-37

[4]. Powell, J. H., and Roberts, J. H. T., "On the frequency of Vibration of CircularDiaphragms", Proceedings of the Physical Society, London, Vol. 35, pp. 170-182, 1923

[5]. McLachlan N. W., "The accession to inertia of flexible discs vibrating in a fluid",Proceedings of the Physical Society of London, vol. 44, pp. 546-555, 1932

[6]. Kwak, M. K. and Kim, K. C., "Axisymmetric vibration of circular plates in contact with fluid",Journal of Sound and Vibration, vol. 146(3), pp.381-389, 1991

[7]. Kwak, M. K., "Vibration of circular plates in contact with water", Journal of AppliedMechanics, ASME, vol. 58, pp. 480483, 1991

[8]. Kwak, M. K., "Hydroelastic vibration of rectangular plates", Journal of Applied Mechanics,ASME, vol. 63, pp. 110115,1996

[9]. Watanabe E, Utsunomiya T. Transient response analysis of a VLFS at airplane landing. In: Watanabe E, editor. Proc International Workshop on Very Large Floating Strutures, Hayama, Kanagawa, Japan, November 25-28. 1996, p. 24-7.

[10]. Watanabe E, Utsunomiya T, Tanigaki S. A transient response analysis of a very large floating structure by finite element method Structural Engrg/Earthquake Engrg, JSCE 1998;15(2): 155s-163s.

[11]. Miao Q, Du S, Dong S, Wu Y. Hydrodynamic analysis of a moored very large floating structure. In: Watanabe Y, editor. ProcIntWorkshopo n Very Large Floating Structures, Hayama, Kanagawa, Japan, November 25-28.1996, p. 201-8.

[12]. Endo H, Yago K, Chiaki S. Elastic responses of a floating platform stimulated by dynamic load. Proc 14th Ocean EngSymp, SocNav Arch Japan. 1998, p. 411-6.
[13]. Ohmatsu S. Numerical calculation of hydroelastic behaviour of VLFS in time domain. Proc 2nd Int Conf. Hydroelastic MarineTech, Fukuoka. 1998, p. 89-97.

[14]. Endo H. The behaviour of a VLFS and an airplane during takeoff/landing run in wave condition. Marine Structures 2000; 13: 477-91.

[15]. Kashiwagi M. A time-domain mode-expansion method for calculating transient elastic responses of a pontoon- type VLFS. JMarine SciTechnol 2000; 5: 89-100. 